\title{
Full speed AHEAD in antibody discovery
}

\author{
Current antibody discovery technologies are limited in terms of their efficacy, accessibility, and scalability. \\ The AHEAD system addresses these limitations by presenting an accelerated evolution workflow for antibody \\ engineering that combines autonomous gene diversification with protein display technology.
}

Paul R. Sargunas and Jamie B. Spangler

A ntibodies have established themselves as a critically important class of proteins in the biomedical sciences. Thanks to their ability to bind specifically and tightly to different biomolecules, antibodies have become invaluable tools for basic biological research, clinical diagnostics, and therapeutic treatments. There are two main approaches by which new antibodies are discovered: animal immunization, in which an animal is infected with an antigen of interest to generate antibodies via an immune response; and protein surface display platforms, which allow the presentation and efficient screening of large libraries of protein variants on the surface of phage, yeast, or other cell types. However, these approaches are limited with respect to their efficacy, speed, and scalability. In a new study, Wellner et al. ${ }^{1}$ addressed these limitations by developing a synthetic recombinant antibody generation technology that integrates an orthogonal DNA replication system (OrthoRep) $)^{2}$ with yeast surface display $^{3}$ to allow continuous gene mutation and accelerated antibody evolution.

Animal immunization has been used for over 100 years to generate therapeutic monoclonal antibodies ${ }^{4}$. Animal-derived antibodies benefit from undergoing in vivo affinity maturation via the process of somatic hypermutation, which enables the development of high-affinity clones from initial low-affinity germline antibodies through continuous evolution. Nonetheless, animal immunization techniques suffer from a variety of fundamental drawbacks, including the requirement for antigens to be immunogenic, biased representation of particular clones (immunodominance), and animal ethics concerns. The development of in vitro surface display technologies has overcome many of these shortcomings, enabling identification of affinity reagents to non-immunogenic antigens, allowing for the design of unbiased evolutionary schemes, and obviating the need for animal use in antibody generation ${ }^{5,6}$. These improvements, however, come at a cost: surface display
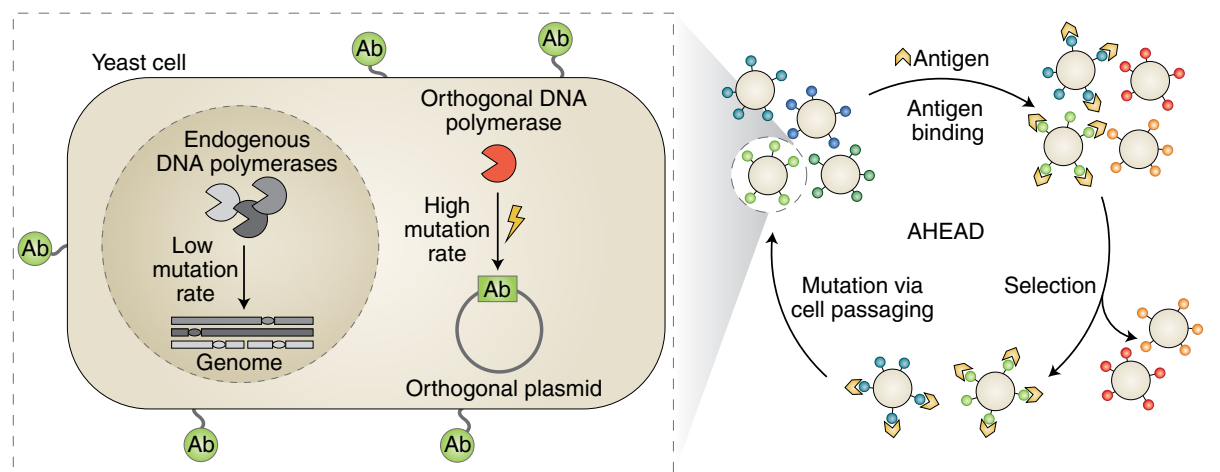

Fig. 1 AHEAD system for continuous hypermutation and accelerated evolution of antibodies. In the AHEAD platform, yeast present antibody ( $A b)$ fragments generated using an orthogonal DNA replication system, which introduces mutations at a much higher rate than endogenous yeast DNA polymerases responsible for replicating the genome (left). Through rounds of enrichment for antigen-binding clones via FACS selections and autonomous mutation of antibody sequences during cell passaging (right), the AHEAD system presents a streamlined process to engineer high-affinity clones.

technologies forfeit the continuous evolution process of somatic hypermutation, discretizing the affinity maturation process. As animal immunization and surface display have become increasingly sophisticated technologies, the barrier to entry has grown for many researchers who would benefit from the discovery of high-quality antibodies against custom molecular targets.

Building on exciting advances in the protein engineering field, Wellner et al. sought to develop an accelerated evolutionary framework for antibody discovery that synthesizes OrthoRep with yeast surface display technology. The key advantage of the OrthoRep system lies in its ability to manipulate the mutation rate of a specific plasmid within the yeast cell (known as p1) without influencing the mutation rate of the yeast cell genome. The error rate in replicating the 1 plasmid is 100,000 -fold higher than that of the yeast genome, leading to targeted diversification of the gene of interest without compromising fitness of the yeast cell host. The platform developed by Wellner et al. ${ }^{1}$, known as autonomous hypermutation yeast surface display (AHEAD), entails iterative rounds of evolution with minimal user intervention. The antibody of interest is encoded on the pl plasmid to allow continuous mutagenesis, and fluorescence-activated cell sorting (FACS) selections are interspersed with generations of yeast cell growth to orchestrate the enrichment of high-affinity antibodies against the desired target protein (Fig. 1).

The AHEAD system was initially applied to affinity mature a single-domain antibody (nanobody) targeting a G-protein-coupled receptor (GPCR) and achieved 20 -fold affinity enhancement. Interestingly, although some affinity-boosting mutations observed with the AHEAD workflow overlapped with those found using alternative evolutionary approaches ${ }^{7}$, others were unique to AHEAD. After revamping the 1 plasmid to bolster yeast expression of encoded antibodies, the investigators challenged their platform to mature, in parallel, eight nanobodies that bound to the receptor binding domain of the severe acute respiratory syndrome coronavirus 2 (SARS-CoV-2) spike protein. All eight selection streams 
yielded nanobodies with superior affinity compared to their respective parents, and improvements of up to 580 -fold were achieved. The functional improvements in terms of SARS-CoV-2 pseudovirus neutralization were even more dramatic, with up to 925 -fold increases in potency. Yet more striking was the observation that the strongest neutralizing clones derived from relatively weak parents, suggesting that the diverse representation of clones afforded by the AHEAD system can lead to the selection of more functionally active progeny that could be missed in workflows that do not accommodate parallel evolutionary streams.

Foreshadowing the promise of their new system in end-to-end antibody development, Wellner et al. ${ }^{1}$ adapted a $2 \times 10^{5}$ member nanobody library ${ }^{8}$ onto the AHEAD platform and performed a successful discovery and affinity maturation campaign. This exciting proof of concept establishes compatibility of their technique with isolating binders from a naïve pool of antibodies and affinity maturation of these clones within the same selection scheme. Moreover, encoding a naïve library on AHEAD demonstrates versatility of the platform and suggests that this approach could be implemented for a variety of other binding scaffolds and protein templates. Indeed, establishing a panel of ready-made libraries for identifying and refining target-specific proteins has vast potential for making the molecular discovery process more accessible and effective. Further iterations of the AHEAD system will likely expand upon the diversity and format of encoded libraries, as well as introduce new selection schemes and/or enzymes to manipulate the evolutionary process.

In vitro evolution has recently commanded the spotlight with the 2018 Nobel Prize in Chemistry, shared by Frances Arnold for directed evolution of enzymes and George Smith and Sir Gregory Winter for phage display of peptides and antibodies. Development of continuous evolution technologies has the potential to catapult the next generation of in vitro evolution workflows. Since the groundbreaking study by Wright and Joyce in 1997, which continuously evolved RNA ligase ribozymes", considerable effort has been invested in establishing methodologies to parallelize and minimize user intervention in the selection process. The advent of phage-assisted continuous evolution (PACE) represented a tremendous advance for enzyme engineering, enabling autonomous protein evolution by coupling the desired protein function to the production of infectious phage ${ }^{10}$. In similar fashion, the AHEAD platform establishes a continuous hypermuation workflow for antibody discovery using an in vitro display system. The wide-ranging possibilities that emerge from this technology promise revolutionary advances in the field of protein engineering.

Paul R. Sargunas (iD) 1 and

Jamie B. Spangler (D) 1,2,3,4,5,6,7凶

${ }^{1}$ Department of Chemical \& Biomolecular

Engineering, Johns Hopkins University, Baltimore,
MD, USA. ${ }^{2}$ Department of Biomedical Engineering, Johns Hopkins University, Baltimore, MD, USA. ${ }^{3}$ Translational Tissue Engineering Center, Johns Hopkins University, Baltimore, MD, USA. ${ }^{4}$ Department of Oncology, Johns Hopkins University, Baltimore, MD, USA. ${ }^{5}$ Bloomberg Kimmel Institute for Cancer Immunotherapy, Johns Hopkins University, Baltimore, MD, USA. ${ }^{6}$ Sidney Kimmel Comprehensive Cancer Center, Johns Hopkins University, Baltimore, MD, USA. ${ }^{7}$ Department of Ophthalmology, Johns Hopkins University, Baltimore, $M D$, USA.

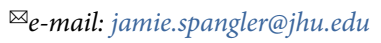

Published online: 1 July 2021

https://doi.org/10.1038/s41589-021-00838-y

References

1. Wellner, A. et al. Nat. Chem. Biol. https://doi.org/10.1038/s41589021-00832-4 (2021).

2. Ravikumar, A., Arzumanyan, G. A., Obadi, M. K. A., Javanpour, A. A. \& Liu, C. C. Cell 175, 1946-1957.e13 (2018).

3. Boder, E. T. \& Wittrup, K. D. Nat. Biotechnol. 15, 553-557 (1997).

4. Köhler, G. \& Milstein, C. Nature 256, 495-497 (1975).

5. Laustsen, A. H. et al. Trends Biotechnol. https://doi.org/10.1016/j. tibtech.2021.03.003 (2021).

6. Gray, A. et al. Nat. Biotechnol. 38, 1234-1239 (2020).

7. Wingler, L. M., McMahon, C., Staus, D. P., Lefkowitz, R. J. \& Kruse, A. C. Cell 176, 479-490.e12 (2019).

8. Shin, J. E. et al. Nat. Commun. 12, 2403 (2021).

9. Wright, M. C. \& Joyce, G. F. Science 276, 614-617 (1997).

10. Esvelt, K. M., Carlson, J. C. \& Liu, D. R. Nature 472, 499-503 (2011).

\section{Acknowledgements}

This work was supported by an E. Matilda Ziegler

Foundation for the Blind Award to J.B.S. P.R.S. is supported by a National Institutes of Health Training Grant Fellowship (T32EY007143) and a National Science Foundation Graduate Research Fellowship Program award.

Competing interests

The authors declare no competing interests.

\title{
Split-personality inhibitors
}

\author{
A rationally designed class of $\mathrm{mTORC} 1$ inhibitors combines the selectivity of rapamycin with the potency of mTOR \\ kinase inhibitors.
}

\section{Carson C. Thoreen}

T he mTOR kinase is a major regulator of cell growth and metabolism and is also hyperactive in most cancers. These features have spurred many efforts to target mTOR therapeutically ${ }^{1}$. Currently available mTOR inhibitors fall into two classes: analogs of the classical allosteric mTOR inhibitor rapamycin termed 'rapalogs' and ATP-competitive mTOR kinase inhibitors (mTORKIs) that directly target mTOR kinase functions (Fig. 1) $)^{2}$. To date, both classes have yielded underwhelming results in the clinic, as rapalogs often lack potency and mTORKIs suffer from toxicity. In this issue, Lee et al. use a clever strategy to engineer a new class of inhibitors that combines the advantages of rapalogs and mTORKIs while mitigating key drawbacks ${ }^{3}$.

Rapalogs are the most widely used class of mTOR inhibitors. Like rapamycin (Fig. 1), they employ an unusual allosteric mechanism that involves binding to both mTOR and a second protein called FKBP12. 